\title{
An Omnidirectional and Low-VSWR Antenna for the FCC-Approved UWB Frequency Band*
}

\author{
Takuya Taniguchi* and Takehiko Kobayashi \\ Wireless Systems Laboratory, Tokyo Denki University \\ 2-2 Nishiki-cho. Kanda, Chiyoda-ku, Tokyo, 101-8457 Japan \\ Phone/Fax: +81-3-5280-3839 E-mail: t-tani@grace.c.dendai.ac.jp \\ I. INTRODUCTION
}

Recently, encouraged by deregulation ultra-wideband (UWB) technology has attracted attention for use in communication and sensing applications in the commercial domain. In February 2002 the Federal Communications Commission (FCC) in the United States conditionally waived unlicensed operation of the personal UWB products in the private sector. In the meantime Various antennas have been developed to be used for UWB systems, such as double-ridged waveguide horn. log-periodic, biconical, and monocone antennas. None of their antennas, however, simultaneously meet omnidirectional and low voltage standing wave ratio (VSWR) requirements, essential for some measurement applications such as UWB channel sounding. Double-ridged waveguide horn and log-periodic antennas are directional. Infinite biconical antennas (Fig. I(a)) have a self-similar structure and are, therefore, frequency-independent, and omnidirectional in azimuth. By replacing one of the cones with the infinite ground plane, the infinite biconicals are extended to infinite monocones (Fig. l(b)), which are also frequency-independent and omnidirectional. By truncating the ideal infinite monocone, a real finite monocone (Fig. 1(c)), widely known as a discone, can be obtained. This structure is omnidirectional in azimuth, but not frequency-independent anymore since the truncation causes reflected current from the base of the cone. A volcano smoke antenna [1] (Fig.1 (d)) fulfills these two requirements simultaneously, but its shape is not well-defined.

We proposed an antenna that is both omnidirectional in azimuth and nearly frequency-independent and defined as a combination of simple structure [2]. Since the proposal was made before the FCC's approval, the first prototype antenna was calculated and constructed to minimize the maximum VSWR between 2 and $10 \mathrm{GHz}$. This paper

\footnotetext{
- This work has becn done partially on a grant for Top Priority Rescarch and Development to be Focused (Frequency Resources Development) from the Ministry of Public Management. Home Affairs, Posts and Telecommunications of Japan within its R \& D Support Scheme for Funding Selected IT Proposals.
} 


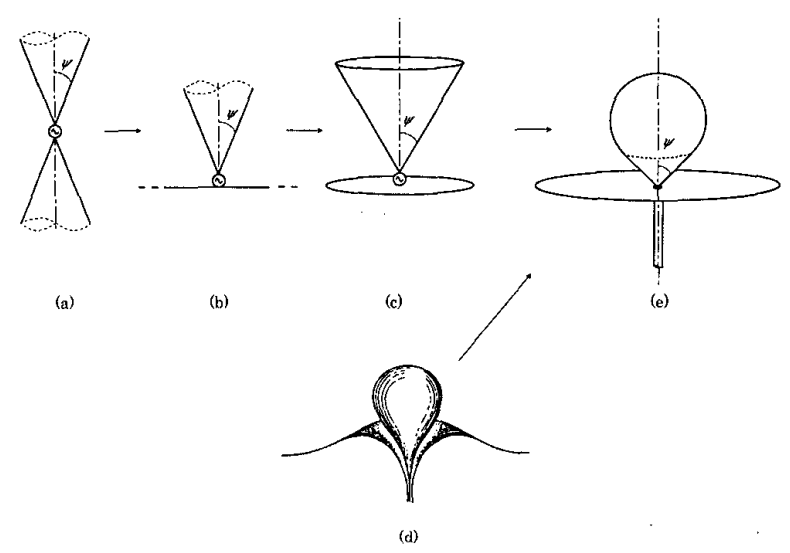

Fig. 1. Broadband omnidirectional antennas: (a) infinite biconical, (b)infinite monocone (c)finite monocone (discone), (d) volcano smoke (in cutaway view) [1], and (e) proposed antenna.

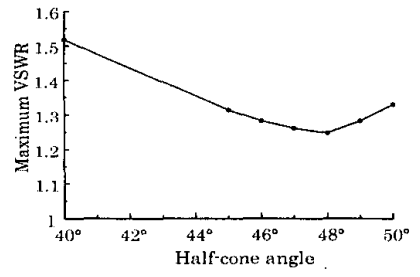

Fig. 2. Computed maximum VSWR of the proposed antenna between 3.1 and $10.6 \mathrm{GHz}$

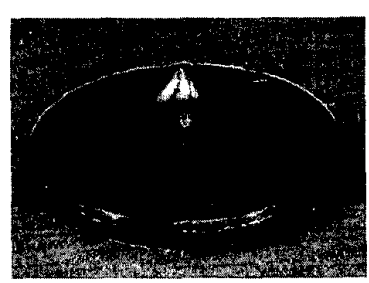

Fig. 3. Prototype antenna.

reports on our second prototype antenna which is optimized in the FCC-approved UWB frequency band ( 3.1 to $10.6 \mathrm{GHz}$ ).

II. The Proposed Antenna and Itsis Optimization

The proposed antenna consists of a circular ground plane and a teardrop that is defined as a combination of a finite cone and a sphere to be inscribed inside the cone at the cone's base, as shown in Fig. 1(e). The teardrop is excited with a coaxial cable penetrating the ground plane. This antenna is considered either as a rounded finite monocone antenna (Fig. 1(c)) or as a simplified form of the Volcano smoke (Fig. 2(d)). Input impedances of the infinite biconical and infinite monocone antennas are governed by the half-cone angle $\psi[1]$. It is also expected that the input impedance of the proposed 


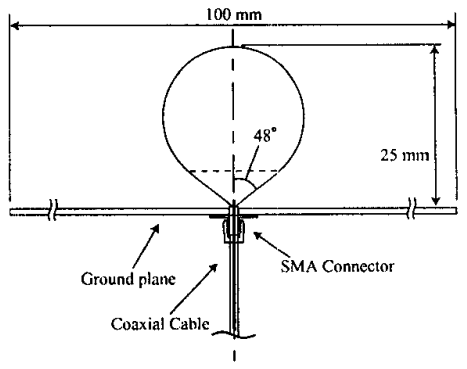

Fig. 4. Cross Section of prototype antenna.

antenna is determined by the half-cone angle $\psi$. We computed the input impedance using CST MICROWAVE STUDIO ${ }^{\text {TM }}$ based on the finite integration method [3], suitable for calculating wideband radiation characteristics from curved structures. Assuming $50-\Omega$ excitation, the maximum VSWR between 3.1 and $10.6 \mathrm{GHz}$ is shown in Fig. 2 , from which the optimum half-cone angle $\psi$ is found $48^{\circ}$. This formation was found to outperform the finite monocone in the VSWR through calculation and the first prototype experiment [2].

We constructed the second prototype antenna as shown in Fig. 3 and 4. The teardrop is $25 \mathrm{~mm}$ high and ground plane is $100 \mathrm{~mm}$ in diameter. The minimum height of the teardrop was calculated to be around a quarter wavelength of the lowest operation frequency.

\section{Measured and Calculatfod Results}

Measurements of the prototype antenna were carried out in a 3-m radio anechoic chamber with use of a microwave network analyzer. The VSWR and radiation patterns were calculated with CST MICROWAVE STUDIO ${ }^{\mathrm{TM}}$. The measured and calculated VSWRs are shown in Fig. 5. The prototype antenna demonstrated VSWR $<1.4$ between 3 and $20 \mathrm{GHz}$.

The measured and calculated E-plane radiation patterns of the proposed antenna at $3,6,9$ and $12 \mathrm{GHz}$ are presented in Figs. 6 and 7, where $0^{\circ}$ corresponds to the zenith and $90^{\circ}$ and $270^{\circ}$ correspond to the horizontal plane, if the antenna is placed like Figs. 3 and 4. This antenna radiates the maximum power in the region from $30^{\circ}$ to $70^{\circ}$. The maximum gain is $3 \pm 4 \mathrm{dBi}$ (measured) and $4 \pm 1 \mathrm{dBi}$ (calculated) and at $90^{\circ}$ (or $270^{\circ}$ ) a gain $-2.5 \pm 1.5 \mathrm{dBi}$ (measured) and $-2 \pm 2 \mathrm{dBi}$ (calculated). These values are in a reasonable agreement, taking into account at least $\pm 1.5 \mathrm{~dB}$ uncertainty in gain measurement $( \pm 1 \mathrm{~dB}$ of a double-ridged waveguide horn as a standard gain antenna and $\pm 0.5 \mathrm{~dB}$ in the microwave network analyzer). To minimize the uncertainty, a 3-antenna technique should be employed. 


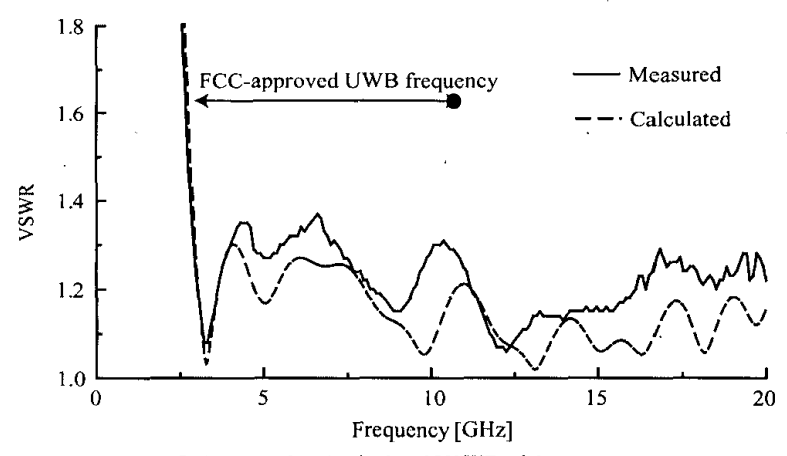

Fig. 5. Measured and calculated VSWR of the prototype antenna.

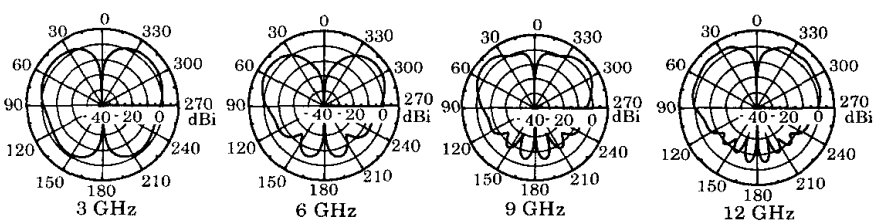

Fig. 6. Calculated E-plane radiation patterns of the prototype antenna.

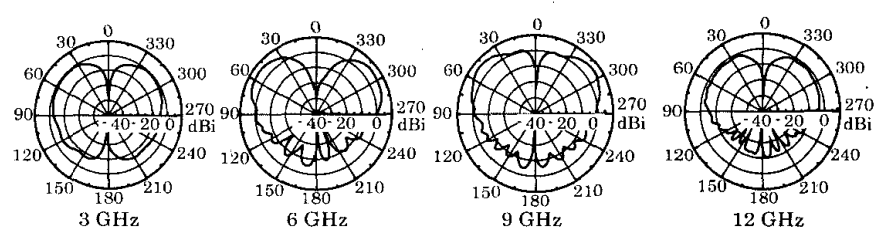

Fig. 7. Measured E-plane radiation patterns of the prototype antenna.

IV. Concuusion

To improve on previous models we constructed a prototype antenna for the FCCapproved UWB frequency band $(3.1-10.6 \mathrm{GHz})$. The prototype antenna was successful because it demonstrated omnidirectional radiation and low VSWR $(<1.4)$ between 3 $\mathrm{GHz}$ and $20 \mathrm{GHz}$. This prototype antenna could therefore be used in the next generation of UWB wireless system measurement applications.

\section{REFERENCE}

[1] J. D. Kraus, Antennas, 2nd edition, McGraw-Hill, New York, 1988, pp. 692-694.

[2] T. Taniguchi and T. Kobayashi, "An Omnidirectional and Low-VSWR Antenna for UltraWideband Wireless Systems," 2002 IEEE Radio and Wireless Conf., pp. 145-148, Aug. 1I-14, 2002.

[3] T. Barts, et al., "Maxwell's grid equations," Frequenz. vol. 44, no. 1, pp. 9-16. 1990. 